\title{
Breaching the barrier
}

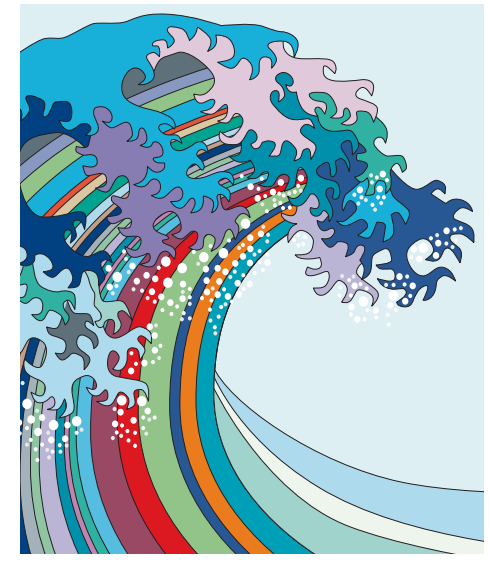

New research in Nature Medicine indicates that the systemic immune activation that is characteristic of chronic HIV infection could be caused by the translocation of microorganisms through a breach in the integrity of the mucosal barrier in the gut.

HIV replicates preferentially in mucosal tissues such as the gut, as these areas are rich in $\mathrm{CD} 4^{+} \mathrm{T}$ cells that express the HIV co-receptor CC-chemokine receptor 5 (CCR5). It was recently determined that the most marked depletion of $\mathrm{CD}^{+}{ }^{+} \mathrm{CCR}^{+} \mathrm{T}$ cells in HIV infection occurs in the gut during acute infection. This led to the hypothesis that this breach in the integrity of the mucosal immune system, perhaps in combination with damage to the intestinal epithelium, could allow the translocation of microorganisms from the intestinal lumen, leading to the systemic immune activation seen during chronic HIV infection. Brenchley et al. now present experimental evidence to support this hypothesis.

The levels of lipopolysaccharide (LPS) in plasma can be used to quantify the extent of microbial translocation. The authors found that the plasma levels of LPS were significantly higher in HIV-infected than in uninfected individuals. To determine the main source of LPS, a simian immunodeficiency virus (SIV) infection model was used and SIV-infected macaques were treated with antibiotics for two weeks. The antibiotic treatment decimated the faecal bacterial load, which correlated with a significant decrease in plasma LPS, indicating that the source of the plasma LPS is likely to be microorganisms from the gastrointestinal tract.

Does this LPS have an immunostimulatory effect in vivo? Assessment of the levels of two plasma proteins known to be induced by LPS provided evidence that it does, as the levels of both proteins were higher in HIV-infected than uninfected individuals. Additionally, increased plasma LPS levels showed a positive correlation with several measures of innate and adaptive immune stimulation. Brenchley et al. went on to investigate the effects of highly active antiretroviral therapy (HAART) and found that after 48 weeks on a HAART regimen, the plasma LPS levels decreased, indicating that a reduction in HIV replication is associated with the restoration of mucosal immune protection in the gut. Finally, sooty mangabeys are natural hosts for SIV and chronic SIV infection in these monkeys is non-pathogenic. SIV-infected sooty mangabeys show low plasma LPS levels, indicating that in this benign infection microbial translocation does not occur.

These data are consistent with a model in which the continuous replication of HIV during chronic infection prevents the repair of the breach in the gut mucosal barrier that occurs during acute infection. This allows microorganisms to translocate from the intestinal lumen and cause systemic immune activation. The authors conclude by suggesting that these findings could lead to the development of new HIV therapeutics.

Sheilagh Molloy Senior Editor, Nature Reviews Microbiology

ORIGINAL RESEARCH PAPER

Brenchley, J. M. et al. Microbial translocation is a cause of systemic immune activation in chronic HIV infection. Nature Med. 19 November 2006

(doi:10.1038/nm1511)

\section{IN BRIEF}

\section{$\Rightarrow$ T CELLS}

Gfi-1 plays an important role in IL-2-mediated Th2 cell expansion.

Zhu, J. et al. Proc. Natl Acad. Sci. USA 103, 18214-18219 (2006)

The transcriptional repressor growth-factor independent 1 (GFI1) has been implicated in promoting clonal expansion of Thelper $2\left(\mathrm{~T}_{\mathrm{H}} 2\right)$ cells in response to interleukin-2 (IL-2). This has now been confirmed by the generation of conditional knockout mice that lack Gfi1 in CD4 ${ }^{+} \mathrm{T}$ cells. CD4 ${ }^{+} \mathrm{T}$ cells from conditional $\mathrm{Gfi}^{-1-}$ mice developed normally but, when placed in $\mathrm{T}_{\mathrm{H}} 2$-cellpolarizing conditions in vitro, the clonal expansion of $\mathrm{T}_{\mathrm{H}} 2$ cells was impaired. This defect also occurred in vivo, as fewer $\mathrm{T}_{\mathrm{H}} 2$ cells developed in conditional Gfi ${ }^{-1-}$ mice than in control mice after infection with Schistosoma mansoni. Consistent with a role for GFI1 in IL-2-mediated proliferation, the activation of STAT5 (signal transducer and activator of transcription 5) by IL-2 was reduced in Gfi1 ${ }^{-1-}$ T cells. However, overexpression of STAT5 in $\mathrm{Gfi}^{-/-} \mathrm{T}$ cells did not rescue the proliferation defect, indicating that GFI1 functions downstream of, or in parallel with, STAT5 signalling to achieve optimal $\mathrm{T}_{\mathrm{H}} 2$-cell expansion.

\section{MMUNOTHERAPY}

An essential role for Akt 1 in dendritic cell function and tumor immunotherapy.

Park, D. et al. Nature Biotech. 3 December 2006 (doi:10.1038/nbt1262) Current dendritic cell (DC)-based vaccines for tumour therapy often fall short because the transfused DCs tend to be shortlived and only transiently active. So, the authors sought ways to improve DC survival and maturation, so that better T-cell responses could be induced. When DCs were deprived of GM-CSF (granulocyte/macrophage colony-stimulating factor), the amount of AKT protein declined before DC death. Exposure of the DCs to lipopolysaccharide and CD40-specific antibody restored AKT expression and prolonged DC survival. The role for AKT in supporting DC survival and maturation was confirmed by expressing a constitutively active, lipid-raft-targeted form of AKT $\left(M_{F}-\triangle A K T\right)$. Transfer of $M_{F}-\Delta$ AKT-expressing DCs into mice bearing established tumours led to robust and long-lasting tumour-specific T-cell responses that eliminated the tumours. So, enhancing DC survival by manipulating AKT might prove beneficial for future strategies of tumour immunotherapy.

\section{AUTOIMMUNITY}

Responses against islet antigens in NOD mice are prevented by tolerance to proinsulin but not IGRP.

Krishnamurthy, B. et al. J. Clin. Invest. 116, 3258-3265 (2006)

Type 1 diabetes is characterized by immune responses to several autoantigens expressed by pancreatic $\beta$-cells, including proinsulin and IGRP (islet-specific glucose-6-phosphatase catalytic-subunit-related protein). But do immune responses to the individual proteins develop independently or does a response spread from one protein to another? To study this, the authors assessed the specificity of T cells arising in non-obese diabetic (NOD) mice that were made tolerant to proinsulin by overexpressing proinsulin-2 in antigen-presenting cells. These mice failed to mount T-cell responses to both proinsulin and IGRP, and this protected the mice from the development of insulitis. By contrast, mice overexpressing IGRP failed to mount responses to IGRP but still generated responses to proinsulin and developed insulitis, providing direct evidence that the immune response spreads from proinsulin to IGRP. 